approval for the study was obtained from PMRC ethics review board in Pakistan.

Results Overall, weighted prevalence of HIV among PWID was 38.4\% (95\% CI; 37.9, 38.9), 7.1\% (95\% CI; 6.8, 7.4) among TGs, 5.4\% (95\% CI: 5.2, 5.6) among MSM and 2.2\% (95\% CI: 2.1, 2.3) among FSWs. Among key HIV risk behaviors condom use at last sex was $27.7 \%$ in TGs, $50 \%$ in FSWs, 24\% in MSM and $15.8 \%$ in PWID, who also shared syringes extensively (31\%). Approximately 5\% of TG, 16.9\% of FSW and $4.0 \%$ of MSM reported having sex with a PWID in past year. Only 24.6\% PWID, 15\% TGs, 8.3\% FSWs and $13.3 \%$ MSM utilized HIV prevention programs in the past year, which explains the escalating trends of HIV among these populations.

Conclusion The HIV epidemic in Pakistan is growing rapidly and is following a rapidly propagating Asian HIV epidemic pattern. Pakistan needs to quickly scale-up services for key populations to contain the epidemic and to prevent a further spread to other KP members and into general population.

Disclosure No significant relationships.

\section{P141 FACTORS ASSOCIATED WITH HIV-RELATED STIGMA AMONG INDIVIDUALS ACCESSING ANTIRETROVIRAL THERAPY IN BRITISH COLUMBIA, CANADA}

${ }^{1}$ Andrea Bever*, 'Sean Grieve, 'Lu Wang, 'William Chau, 'Taylor Mclinden, ${ }^{1}$ Tim Wesseling, ${ }^{1}$ Kate Salters, ${ }^{2}$ Brittany Bingham, 'David Moore, ${ }^{1}$ Rolando Barrios. ${ }^{1} B C$ Centre for Excellence in HIVIAIDS, Vancouver, Canada; ${ }^{2}$ Vancouver Coastal Health, Aboriginal Health, Vancouver, Canada

\subsection{6/sextrans-2019-sti.309}

Background Despite public health messaging that antiretroviral therapy (ART) has improved health outcomes for people living with HIV (PLWH) and is effective in preventing HIV transmission, many PLWH continue to experience HIV-related stigma. It is critical to assess HIV-related stigma experienced by PLWH accessing ART in the modern HIV treatment era.

Methods The STOP HIV/AIDS Program Evaluation (SHAPE) study is a longitudinal cohort of PLWH $\geq 19$ years of age in British Columbia, Canada. This cross-sectional analysis uses SHAPE baseline survey data (collected January 2016-August 2018) and linked clinical registry data to examine factors associated with HIV-related stigma among individuals accessing ART. HIV-related stigma was self-reported using the ten-item Berger HIV stigma scale. Multivariable linear regression quantified the relationship between key explanatory variables and stigma.

Results Among 627 participants, 136(22\%) identified as women, $326(52 \%)$ were aged $\geq 50$ at enrolment, 374(60\%) identified as men who have sex with men, and 133(21\%) selfreported Indigenous ethnicity. The median stigma score was 47.5 (Q1-Q3: 32.5-62.5; range: 0-100). In the multivariable model, reporting injection drug use (IDU) in the past year $(\beta=4.54,95 \% \mathrm{CI}=0.23,8.86)$ or selecting "prefer not to answer" when asked about IDU history $(\beta=9.52,95 \% \mathrm{CI}=$ $4.77,14.28)$; experiences of lifetime violence $(\beta=7.62,95 \%$ $\mathrm{CI}=3.67,11.56)$; and having a mental health disorder diagnosis $(\beta=5.30,95 \% C I=1.88,8.73)$ were associated with higher stigma scores. Higher stigma scores were also associated with being 40-49 years old ( $\beta=6.21,95 \% \mathrm{CI}=1.58,10.85)$ compared to $<40$; age $\geq 50$ had no significant association. Living in a city with a population $\geq 100,000 \quad(\beta=-4.66,95 \% \mathrm{CI}=-$ $8.53,-0.78)$ was associated with lower stigma scores.

Conclusion Age, city size, IDU experience, violence, and mental illness were independently associated with HIV-related stigma. These findings provide support for an intersectional investigation into how these factors propagate stigma and how this experience impacts the health and wellbeing of PLWH in this setting.

Disclosure No significant relationships.

\section{P142 CHARACTERISTICS OF HIV-1 PRETREATMENT DRUG RESISTANCE AND ITS IMPACT ON COMBINED ANTIRETROVIRAL THERAPY IN BEIJING}

${ }^{1}$ Ruolei Xin*, ${ }^{1}$ Chun Huang, ${ }^{1}$ Lishi Bai, ${ }^{1}$ Hongyan Lu, ${ }^{2}$ Lijun Sun, ${ }^{2}$ An Liu. ${ }^{1}$ Beijing Center for Disease Prevention and Control, Beijing, China; ${ }^{2}$ Beijing You'an Hospital, Beijing, China

\subsection{6/sextrans-2019-sti.310}

Background From 2013, the expanded strategies for the initial of combined antiretroviral therapy (cART) were administrated in Beijing to the infected individuals whatever their CD4 cell counts, hence, the increasing trend of HIV-1 epidemic has been alleviated. Pretreatment drug resistance was monitored, yet its impact on cART and the derivation of acquired drug resistance should be well delineated.

Methods Treatment-naïve individuals with HIV-1 were recruited from September 2012 to April 2013 in Beijing You'an Hospital. The patients were followed up after the initial of cART. HIV-1 pol gene fragment was amplified using One-Step RT-PCR at stage of pretreatment and follow-up virological failure. The genotypic drug resistance was interpreted with Stanford University HIV Drug Resistance Database, with CPR algorithm and HIVdb program. The genotypes were determined using MEGA6.0 after multiple alignment by gene cutter, with reference to BLAST and RIP.

Results Totally, 324 treatment-naïve infections were recruited, and about 272 cases (84.0\%) were infected via MSM. The top three subtypes or CRFs were CRF01_AE (60.5\%), CRF07_BC (18.5\%) and B (15.4\%). 13 individuals were observed to possess PDR (4.0\%), at a low prevalence. The accumulative rate of virological failure was 9.5\%, which were observed in 31 cases, and the cohort maintenance rate of $75.4 \%$. The PDR induced virlological failure to develop acquired drug resistance (ADR) in three individuals, at a contributive rate of $23.1 \%$. Some persons gained virological failure at $0.3-0.6$ years post cART. Lower CD4 cell counts were prone to accompanied with the majority of virolgical failure (23.3\%). The dynamic fluctuation of acquired drug resistance were observed by single genome amplification.

Conclusion There was a low prevalence of transmitted drug resistance in treatment-naïve individuals in Beijing, and the TDR might convey $23 \%$ virological failure. The first-line cART obtained good performance of virological suppression, and the virological failure would be rectified by shift to second-line cART.

Disclosure No significant relationships. 